\title{
Prevalence and temporal trends in antimicrobial resistance of bovine respiratory disease pathogen isolates submitted to the Wisconsin Veterinary Diagnostic Laboratory: 2008-2017
}

\author{
C. L. Holschbach, ${ }^{1 *} \odot$ N. Aulik, ${ }^{2,3} \odot$ K. Poulsen, ${ }^{1,3} \oplus$ and T. L. Ollivett ${ }^{1} \odot$ \\ ${ }^{1}$ Department of Medical Sciences, School of Veterinary Medicine, University of Wisconsin, Madison 53706 \\ ${ }^{2}$ Department of Pathobiological Sciences, School of Veterinary Medicine, University of Wisconsin, Madison 53706 \\ ${ }^{3}$ Wisconsin Veterinary Diagnostic Laboratory, Madison 53706
}

\begin{abstract}
The objective of this study was to describe the prevalence and trends in antimicrobial resistance for bacterial pathogens associated with bovine respiratory disease (BRD) isolated from samples submitted to the Wisconsin Veterinary Diagnostic Laboratory (WVDL). Data were retrospectively collected from bovine respiratory isolates including Pasteurella multocida, Mannheimia haemolytica, Histophilus somni, and Bibersteinia trehalosi identified at the WVDL between January 2008 and December 2017. Antimicrobial susceptibility testing data were queried from antimicrobial resistance databases at the WVDL. A total of 4,261 isolates were identified. Pasteurella multocida was most frequently identified, accounting for 2,094 isolates (49\% of total) over the study period. Mannheimia haemolytica was the second most frequently isolated bacterial respiratory pathogen $(\mathrm{n}=1,267,30 \%)$ followed by $H$. somni $(\mathrm{n}=749,18 \%)$ and $B$. trehalosi $(\mathrm{n}=151,4 \%)$. Over the 10 -yr period, B. trehalosi had the highest median percentage of isolates that were resistant to at least one antibiotic at 33\% (interquartile range: 24,47 ) followed by $M$. haemolytica $(13 \% ; 8,23)$. For P. multocida, $10 \%$ $(4,26)$ of isolates were classified as resistant to at least one antibiotic, whereas $H$. somni had the fewest resistant isolates $(9 \% ; 3,15)$. When comparing 2013-2017 to 2008-2012, the overall percentage of resistant isolates for P. multocida and B. trehalosi decreased, whereas the percentage of resistant isolates for $M$. haemolytica and $H$. somni increased. Increased resistance against florfenicol, fluoroquinolones, gentamicin, tilmicosin, and tulathromycin was observed for $M$. haemolytica. These data show that antimicrobial susceptibility for
\end{abstract}

Received September 10, 2019.

Accepted May 19, 2020.

*Corresponding author: cholschbach@wisc.edu
BRD bacterial pathogens has changed in the population served by the WVDL over this 10-yr period. For P. multocida, resistance is relatively low and has either improved or at least remained constant for the majority of drugs labeled for treatment of respiratory disease in dairy cattle. Veterinarians and producers should be aware of the bacterial pathogens most commonly associated with BRD and work toward early disease detection, proper antibiotic administration, and monitoring lung lesions to ensure that their treatment protocols improve lung health.

Key words: bovine respiratory disease, antimicrobial resistance, dairy, Wisconsin

\section{INTRODUCTION}

Bovine respiratory disease (BRD) is a multifactorial syndrome caused by numerous infectious agents as well as compromised host defenses and environmental conditions (Woolums, 2015). Respiratory disease is a leading cause of morbidity and mortality in preweaned dairy calves, and also negatively affects growth, reproductive performance, milk production, and longevity of adult dairy cattle (Bach, 2011; Teixeira et al., 2017; Dunn et al., 2018). Pasteurella multocida, Mannheimia haemolytica, Histophilus somni, and Mycoplasma bovis are generally accepted as important contributors to respiratory disease in ruminants (Woolums, 2015); however, data regarding the prevalence of these pathogens in cattle on dairy farms are limited. Bibersteinia trehalosi, formerly known as Pasteurella trehalosi, is another bacterial pathogen that may play a primary role in BRD (Confer, 2009).

Because bacterial bronchopneumonia is the most common form of pneumonia seen in dairy calves (Panciera and Confer, 2010), antibiotics are used for both the control and treatment of clinical disease (DeDonder and Apley, 2015). According to the latest National Animal Health Monitoring System dairy study, BRD 
is one of the most common reasons for antibiotic use in preweaned, weaned, and adult dairy cattle (USDA, 2018). Macrolides and florfenicol are the most commonly used antibiotics for the treatment of BRD in preweaned and weaned dairy calves (USDA, 2018). Beta-lactams, namely third-generation cephalosporins, are the mainstay of treatment for adult cattle with BRD (USDA, 2018). Other classes of commonly used antibiotics include fluoroquinolones, tetracyclines, and sulfonamides (USDA, 2018).

Exposure of bacteria to antibiotics has the potential to create selection for resistant organisms (Portis et al., 2012). Bacteria display 1 of 3 resistance phenotypes: susceptible, intrinsic resistance, or acquired resistance (Boerlin and White, 2013). Intrinsic resistance, a form of innate resistance, affects all members of a specific bacterial genus, species, or subspecies (Boerlin and White, 2013). In contrast, antibiotic resistance can be acquired when a susceptible organism develops resistance through genetic modification (Boerlin and White, 2013). Acquired resistance can span from resistance to a single agent within a class to an entire class of antibiotics (Boerlin and White, 2013).

In the dairy industry, detection of resistance to BRD pathogens has been hampered by a lack of surveillance programs and, until recently, a lack of BRD-specific interpretative criteria that allow for detection of resistance (Watts and Sweeney, 2010). Antimicrobial susceptibility testing (AST) is used to determine the activity of certain antibiotics against specific pathogens (Watts and Sweeney, 2010). These tests provide in vitro assessment of antimicrobial activity and help predict clinical response (Watts and Sweeney, 2010). In veterinary medicine, the Clinical Laboratory and Standards Institute (CLSI) provides clinical breakpoints for many but not all pathogen-drug combinations.

Third-generation cephalosporins, fluoroquinolones, and macrolides are drug classes commonly used for BRD treatment, and have been identified as highest priority critically important antimicrobials in human medicine (WHO, 2019). To maintain antibiotic efficacy in veterinary and human patients, producers and veterinarians must practice good antibiotic stewardship (Portis et al., 2012). Effective antibiotic stewardship is in part contingent on surveillance of antibiotic resistance in animals (Valenzuela et al., 2017). Information obtained through antibiotic resistance surveillance can be used to guide optimal antibiotic selection in hope of reducing treatment failure. Thus, the objective of our study was to describe the prevalence and trends in antimicrobial resistance (AMR) for bacterial pathogens associated with BRD isolated from samples submitted to the Wisconsin Veterinary Diagnostic Laboratory (WVDL) over a 10-yr period.

\section{MATERIALS AND METHODS}

\section{Data Collection}

Data were retrospectively collected from bovine respiratory tract isolates including $P$. multocida, $M$. haemolytica, H. somni, and B. trehalosi identified at the WVDL between January 1, 2008, and December 31, 2017, using the Laboratory Information Management System (UVIS, Ross Group Inc., Dayton, OH) and AMR databases at the WVDL Madison and Barron locations (Sensititer, Thermo Fisher Scientific, Waltham, MA). The majority of the WVDL caseload comes from the state of Wisconsin, with approximately $90 \%$ of the samples originating from cattle on dairy farms (Keith Poulsen, personal communication). Only samples obtained from the respiratory tract were analyzed, which included deep nasopharyngeal swabs, bronchoalveolar lavage fluid, nasal swabs, ear swabs, and lung tissue collected at necropsy. Samples submitted from multiple tissues or animals originating from a single farm were grouped onto one accession. In an effort to reduce costs to the clients, the WVDL performed AST on only one isolate of a bacterial pathogen per accession. The bacterial pathogens that underwent AST had been cultured and identified from each sample using standard operating procedures reviewed and accredited by the American Association of Veterinary Laboratory Diagnosticians. Briefly, swabs were cultured on 2 bovine or sheep blood agar, eosin-methylene blue agar, and chocolate agar media plates. One blood agar and the chocolate agar plates were incubated with $5 \% \mathrm{CO}_{2}$ and the others were incubated in aerobic conditions at $36 \pm$ $2^{\circ} \mathrm{C}$ for 18 to $24 \mathrm{~h}$, observed, and incubated another 18 to $24 \mathrm{~h}$ before a second round of observation and identification. Identification was made using MALDI-TOF MS utilized post implementation in November 2014 or various biochemical tests utilized before November 2014.

\section{Antimicrobial Susceptibility}

Isolates had AST performed using the bovine/porcine MIC plate with tulathromycin (Sensititer BOPO6F, Thermo Fisher Scientific, Oakwood Village, OH), which includes 18 antimicrobial agents [ampicillin, ceftiofur, chlortetracycline, clindamycin, danofloxacin, enrofloxacin, florfenicol, gentamicin, neomycin, oxytetracycline, penicillin, sulfadimethoxine, spectinomycin, tiamulin, tilmicosin, trimethoprim-sulfamethoxazole (TMS), tulathromycin, and tylosin]. Additional AST data were obtained on gamithromycin and tildipirosin starting in 2016 using the Kirby-Bauer disk diffusion method. Inclusion of the antibiotics in this study re- 
flects laboratory standards and should not be perceived as an endorsement of their use in cattle.

Antimicrobial susceptibility results included a numerical value corresponding to the MIC and an interpretive criterion of resistant, susceptible, or intermediate based on CLSI standards (CLSI, 2006, 2008, 2013). The interpretative criteria are based on breakpoints of the numerical MIC values, which are developed for specific host-pathogen-drug combinations based on available research (Valenzuela et al., 2017). When CLSI breakpoints were not provided for certain pathogen-drug combinations, breakpoints "supplied by veterinary customer" to Trek Diagnostic Systems were used (Cleveland, $\mathrm{OH})$. These include ampicillin, clindamycin, florfenicol, neomycin, penicillin, spectinomycin, sulfadimethoxine, tiamulin, and tilmicosin as well as tetracycline for $H$. somni. In the event that CLSI guidelines or "supplied by veterinary customer" breakpoints were not published for certain pathogen-drug combinations, MIC values were reported, but results were classified as not interpretable and were not analyzed. For B. trehalosi, no CLSI guidelines were available, so breakpoints were applied using "supplied by veterinary customer." Each drug-pathogen combination was analyzed using the same set of breakpoints throughout the study period. More specifically, data were analyzed using the CLSI 2006 or "supplied by veterinary customer" breakpoints with 2 exceptions regarding tulathromycin and penicillin. For tulathromycin, no CLSI guidelines were available until 2008, the first year of our study. Therefore, the 2008 CLSI guidelines, not the 2006 guidelines, were used to interpret tulathromycin data throughout the study period. In 2015, the WVDL received the 2013 CLSI Database Update, which included the 2013 CLSI breakpoints for penicillin. These breakpoints were different than the previously used "supplied by veterinary customer" breakpoints. Therefore for consistency, we interpreted all of the penicillin data using the 2013 CLSI guidelines. For the purpose of our analyses, susceptible and intermediate categories were combined.

It is important to note there was a specific time point between 2013 and 2018 that tiamulin was not reported at the WVDL and therefore data were not collected or available for analysis. Additionally, in January 2016, Kirby-Bauer disk diffusion was implemented for gamithromycin and tildipirosin. For 4 mo in 2016, the disks were not available, so the number of organisms tested was fewer compared with other antibiotics. Last, upon request, a panel of antimicrobials may have been reported even if one antimicrobial had unsatisfactory results. This is normally due to the wrong concentration or lack of antimicrobial in a particular well. Therefore, the total isolates tested may vary between antimicrobials.

\section{Statistical Analysis}

Yearly numbers of P. multocida, M. haemolytica, $H$. somni, and B. trehalosi isolates were calculated using Microsoft Excel (2019, Microsoft Corp., Redmond, WA). Number and percent of resistant isolates by antibiotic agent in total and over 5 -yr time periods were also tabulated. Group level comparisons of categorical variables were assessed via contingency tables. For cell values $<5$, Fisher's exact test was used to compare proportions. A standard statistical package (SAS v.9.4, SAS Institute Inc., Cary, NC) was used to calculate the relative risk (RR; 95\% CI) of resistance in 2013-2017 versus 2008-2012. If $R R>1$, the likelihood of resistance in 2013-2017 is higher than the likelihood of resistance in 2008-2012. If $R R<1$, the likelihood of resistance in 2013-2017 is lower than the likelihood of resistance in 2008-2012. Significance was set at $\alpha<0.05$.

\section{RESULTS}

A total of 4,261 isolates of P. multocida, M. haemolytica, H. somni, and B. trehalosi were identified at the WVDL between January 2008 and December 2017. Table 1 shows the distribution of BRD bacterial isolates as a number and percentage of total isolates that were tested for antimicrobial susceptibility annually. During each year of the 10-yr study period, P. multocida was the most frequently isolated pathogen from respiratory samples, followed by M. haemolytica, H. somni, and B. trehalosi.

The proportions of resistant isolates as percentages and counts for each BRD pathogen, analyzed by 5-yr time periods, are shown in Tables $2,3,4$, and 5 . The $\mathrm{RR}$ of resistance for each drug-pathogen combination in 2013-2017 compared with 2008-2012 is also shown with associated $P$-values (Tables $2,3,4$, and 5 ). Over the 10-yr study period, B. trehalosi had the highest median percentage of resistant isolates at 33\% (interquartile range 24,47$)$ followed by $M$. haemolytica $(13 \%$; $8,23)$. For P. multocida, $10 \%(4,26)$ of isolates were classified as resistant, whereas $H$. somni had the fewest resistant isolates $(9 \% ; 3,15)$. For P. multocida, M. haemolytica, H. somni, and B. trehalosi, at least one isolate was resistant to each of the antibiotics routinely tested for susceptibility over the 10-yr period.

Five percent or less of all P. multocida isolates were resistant to ceftiofur $(<1 \%)$, enrofloxacin $(2 \%)$, chlortetracycline $(2 \%)$, ampicillin $(2 \%)$, danofloxacin $(4 \%)$, florfenicol (5\%), penicillin (5\%), gentamicin (5\%), and TMS (5\%). Resistance ranged from 8 to $36 \%$ for tulathromycin $(8 \%)$, tiamulin $(12 \%)$, gamithromycin (13\%), spectinomycin (21\%), tildipirosin (23\%), tilmi$\operatorname{cosin}(23 \%)$, and oxytetracycline (36\%). Resistance 


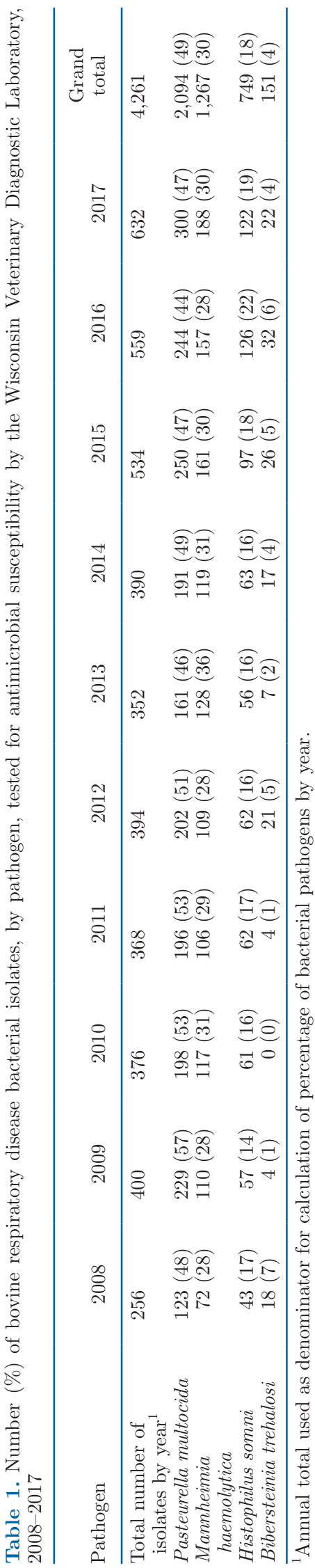

was most common against clindamycin (98\%), tylosin (88\%), neomycin (55\%), and sulfadimethoxine (52\%). Among $P$. multocida isolates detected during the study period, we found a decrease $(P<0.05)$ in resistance to gentamicin, ampicillin, penicillin, florfenicol, tiamulin, TMS, and sulfadimethoxine (Table 2). Resistance to danofloxacin increased $(P>0.05)$, whereas resistance to the remaining antibiotics was unchanged (Table 2).

Less than $10 \%$ of all $M$. haemolytica isolates were resistant to ceftiofur $(<1 \%)$, chlortetracycline $(2 \%)$, tiamulin (2\%), TMS (4\%), florfenicol (7\%), enrofloxacin $(8 \%)$, gentamicin $(8 \%)$, and tulathromycin $(9 \%)$. Resistance ranged from 12 to $40 \%$ for spectinomycin (12\%), gamithromycin (13\%), tildipirosin (13\%), tilmicosin (14\%), ampicillin (16\%), danofloxacin (16\%), penicillin $(21 \%)$, oxytetracycline $(30 \%)$, neomycin $(35 \%)$, and sulfadimethoxine (40\%). Resistance was most common against tylosin (99\%) and clindamycin (98\%). For $M$. haemolytica isolates, we found an increase $(P<0.05)$ in resistance to gentamicin, fluoroquinolones, phenicols, and macrolides (tulathromycin and tilmicosin; Table $3)$. A decrease $(P<0.05)$ in resistance was found to ampicillin and sulfadimethoxine, whereas resistance to the remainder of the antibiotics remained unchanged (Table 3).

For H. somni, $5 \%$ or less of all isolates were resistant to ampicillin (2\%), ceftiofur (2\%), chlortetracycline (3\%), florfenicol (3\%), tiamulin (3\%), TMS (3\%), enrofloxacin (5\%), and tildipirosin (5\%). Resistance ranged from 6 to $50 \%$ for gamithromycin $(6 \%)$, tilmicosin (9\%), tulathromycin (9\%), clindamycin (10\%), tylosin (10\%), penicillin (12\%), spectinomycin (18\%), gentamicin $(22 \%)$, oxytetracycline $(24 \%)$, and sulfadimethoxine (50\%). Resistance was highest for neomycin (78\%). For the majority of $H$. somni isolates, we did not see a change in resistance during the study period. A decrease $(P<0.05)$ in resistance was observed for enrofloxacin, TMS, and sulfadimethoxine (Table 4).

Bibersteinia trehalosi showed the greatest amount of resistance across all antibiotic classes. Aside from tiamulin (8\%) and gentamicin (13\%), resistance ranged from 23 to $100 \%$ for the remaining antibiotics. One hundred percent of $B$. trehalosi isolates were resistant to penicillin, whereas $90 \%$ were resistant to clindamycin. Resistance was also frequently found against oxytetracycline (78\%), sulfadimethoxine (47\%), neomycin $(45 \%)$, tilmicosin (35\%), enrofloxacin (34\%), ampicillin $(32 \%)$, spectinomycin (32\%), chlortetracycline (25\%), TMS (23\%), and florfenicol (23\%). Among B. trehalosi isolates over the 10-yr period, we found a decrease $(P<0.05)$ in resistance to aminoglycosides, fluoroquinolones, macrolides, and sulfonamides, whereas an increase $(P<0.05)$ in resistance to oxytetracycline was observed. 
Table 2. Prevalence (\%, number in parentheses $)^{1}$ of bovine Pasteurella multocida isolates with resistance to different antibiotics based on interpretative categories by 5 -yr increments

\begin{tabular}{|c|c|c|c|c|c|}
\hline Class & Antibiotic & $2008-2012$ & 2013-2017 & $\mathrm{RR}^{2}$ & $P$-value \\
\hline Aminoglycosides & Neomycin & $53(502 / 946)$ & $57(647 / 1,145)$ & 1.06 & 0.12 \\
\hline$\beta$-Lactams & Penicillin & $6(61 / 948)$ & $3(37 / 1,146)$ & 0.50 & $<0.01$ \\
\hline Third-generation cephalosporins & Ceftiofur & $1(6 / 947)$ & $0.01(1 / 1,146)$ & 0.14 & 0.05 \\
\hline Fluoroquinolones & Danofloxacin & $2(20 / 946)$ & $5(60 / 1,145)$ & 2.48 & $<0.01$ \\
\hline \multirow[t]{5}{*}{ Macrolides } & Gamithromycin & - & $13(61 / 471)$ & - & - \\
\hline & Tildipirosin & - & $19(99 / 516)$ & - & - \\
\hline & Tilmicosin & $23(215 / 946)$ & $23(261 / 1144)$ & 1.00 & 0.96 \\
\hline & Tulathromycin & $8(74 / 945)$ & $9(100 / 1,145)$ & 1.12 & 0.46 \\
\hline & Tylosin & $89(840 / 946)$ & $88(1,009 / 1,145)$ & 0.99 & 0.63 \\
\hline Phenicols & Florfenicol & $6(61 / 946)$ & $4(45 / 1,145)$ & 0.61 & $<0.01$ \\
\hline Pleuromutilin & Tiamulin & $16(151 / 945)$ & $4(16 / 401)$ & 0.25 & $<0.01$ \\
\hline
\end{tabular}

${ }^{1}$ Percentage represents resistance as a percentage of $P$. multocida isolates tested against that antibiotic during the 5-yr period.

${ }^{2} \mathrm{RR}=$ relative risk; if $\mathrm{RR}>1$, the likelihood of resistance in 2013-2017 is higher than the likelihood of resistance in 2008-2012. If $\mathrm{RR}<1$, the likelihood of resistance in 2013-2017 is lower than the likelihood of resistance in 2008-2012.

\section{DISCUSSION}

To the authors' knowledge, this is the first report of the prevalence and trends in AMR for P. multocida, $M$. haemolytica, H. somni, and B. trehalosi isolates cultured from respiratory tract samples, originating predominately from cattle on dairy farms, in the upper Mid- west region of the United States. Of these pathogens, $P$. multocida was the most frequently isolated, followed by M. haemolytica, H. somni, and B. trehalosi. The number of respiratory tract isolates analyzed by the WVDL annually has more than tripled since the start of our data collection in 2008, but the distribution of pathogens each year has remained unchanged. Our findings are

Table 3. Prevalence $(\%, \text { number in parentheses })^{1}$ of bovine Mannheimia haemolytica isolates with resistance to different antibiotics based on interpretative categories by 5 -yr increments

\begin{tabular}{|c|c|c|c|c|c|}
\hline Class & Antibiotic & 2008-2012 & $2013-2017$ & $\mathrm{RR}^{2}$ & $P$-value \\
\hline \multirow{2}{*}{ Aminoglycosides } & Neomycin & $38(197 / 513)$ & $33(251 / 753)$ & 0.87 & 0.06 \\
\hline & Spectinomycin & $11(56 / 514)$ & $12(93 / 753)$ & 1.13 & 0.43 \\
\hline$\beta$-Lactams & Penicillin & $23(120 / 514)$ & $19(145 / 753)$ & 0.82 & 0.09 \\
\hline Third-generation cephalosporins & Ceftiofur & $0(0 / 511)$ & $0.66(5 / 753)$ & - & - \\
\hline Fluoroquinolones & Danofloxacin & $12(62 / 514)$ & $19(142 / 753)$ & 1.56 & $<0.01$ \\
\hline \multirow{5}{*}{ Macrolides } & Gamithromycin & - & $13(39 / 291)$ & - & - \\
\hline & Tildipirosin & - & $13(43 / 320)$ & - & - \\
\hline & Tilmicosin & $10(51 / 514)$ & $16(124 / 753)$ & 1.66 & $<0.01$ \\
\hline & Tulathromycin & $6(33 / 511)$ & $11(84 / 753)$ & 1.73 & $<0.01$ \\
\hline & Tylosin & $99(504 / 511)$ & $99(742 / 753)$ & 1.00 & 0.89 \\
\hline Phenicols & Florfenicol & $4(21 / 514)$ & $10(74 / 753)$ & 2.41 & $<0.01$ \\
\hline Pleuromutilin & Tiamulin & $2(9 / 514)$ & $2(6 / 292)$ & 1.17 & 0.76 \\
\hline
\end{tabular}

${ }^{1}$ Percentage represents resistance as a percentage of $M$. haemolytica isolates tested against that antibiotic during the 5-yr period.

${ }^{2} \mathrm{RR}=$ relative risk; if $\mathrm{RR}>1$, the likelihood of resistance in 2013-2017 is higher than the likelihood of resistance in 2008-2012. If $\mathrm{RR}<1$, the likelihood of resistance in 2013-2017 is lower than the likelihood of resistance in 2008-2012. 
Table 4. Prevalence (\%, number in parentheses $)^{1}$ of bovine Histophilus somni isolates with resistance to different antibiotics based on interpretative categories by 5 -yr increments

\begin{tabular}{|c|c|c|c|c|c|}
\hline Class & Antibiotic & 2008-2012 & 2013-2017 & $\mathrm{RR}^{2}$ & $P$-value \\
\hline & Neomycin & $76(216 / 284)$ & $80(365 / 459)$ & 1.05 & 0.27 \\
\hline$\beta$-Lactams & Penicillin & $12(34 / 285)$ & $13(59 / 464)$ & 1.07 & 0.82 \\
\hline Third-generation cephalosporins & Ceftiofur & $1(4 / 283)$ & $2(10 / 458)$ & 1.54 & 0.58 \\
\hline Fluoroquinolones & Enrofloxacin & $7(21 / 284)$ & $3(16 / 458)$ & 0.47 & 0.02 \\
\hline & Tildipirosin & - & $5(10 / 205)$ & - & - \\
\hline & Tilmicosin & $7(19 / 284)$ & $10(48 / 459)$ & 1.56 & 0.08 \\
\hline & Tulathromycin & $7(19 / 284)$ & $10(47 / 458)$ & 1.53 & 0.10 \\
\hline & Tylosin & $10(28 / 285)$ & $10(47 / 464)$ & 1.03 & 0.89 \\
\hline Phenicols & Florfenicol & $2(7 / 284)$ & $3(14 / 459)$ & 1.24 & 0.64 \\
\hline Pleuromutilin & Tiamulin & $1(4 / 282)$ & $5(7 / 142)$ & 3.48 & 0.05 \\
\hline Sulfonamides & Sulfadimethoxine & $55(155 / 284)$ & $47(214 / 459)$ & 0.85 & 0.04 \\
\hline
\end{tabular}

${ }^{1}$ Percentage represents resistance as a percentage of $H$. somni isolates tested against that antibiotic during the 5 -yr period.

${ }^{2} \mathrm{RR}=$ relative risk; if $\mathrm{RR}>1$, the likelihood of resistance in 2013-2017 is higher than the likelihood of resistance in 2008-2012. If $\mathrm{RR}<1$, the likelihood of resistance in 2013-2017 is lower than the likelihood of resistance in 2008-2012. No Clinical and Laboratory Standards Institute breakpoints for danofloxacin.

consistent with that of the most recent extensive survey of bovine respiratory tract bacterial isolates in the United States and Canada, published in 2012 (Portis et al., 2012). Although we cannot specifically determine the pathogen responsible for respiratory disease in each case, it does not appear that there is emergence of BRD associated with less frequently identified BRD pathogens, such as B. trehalosi.

Pathogens analyzed for our study showed greatest resistance against clindamycin, tylosin, and neomycin. Clindamycin is in the class of lincosamide antibiotics, for which there are no formulations labeled for systemic use in cattle (Giguére, 2013a). Tylosin is a macrolide antibiotic primarily used to treat pneumonia associated with Mycoplasma bovis and otitis media and interna in calves (Giguére, 2013b). It is solely labeled for use in beef cattle and nonlactating dairy cattle. With the advent of newer macrolide antibiotics, tylosin is rarely chosen in most clinical situations (Giguére, 2013b). Neomycin is an aminoglycoside used for the oral treatment of enteric infections in calves caused by E. coli; however, increasing resistance to $E$. coli has limited its

Table 5. Prevalence (\%, number in parentheses $)^{1}$ of bovine Bibersteinia trehalosi isolates with resistance to different antibiotics based on interpretative categories by 5 -yr increments

\begin{tabular}{|c|c|c|c|c|c|}
\hline Class & Antibiotic & 2008-2012 & $2013-2017$ & $\mathrm{RR}^{2}$ & $P$-value \\
\hline \multirow{2}{*}{ Aminoglycosides } & Neomycin & $72(34 / 47)$ & $33(34 / 104)$ & 0.45 & $<0.01$ \\
\hline & Spectinomycin & $49(23 / 47)$ & $24(25 / 104)$ & 0.49 & $<0.01$ \\
\hline$\beta$-Lactams & Penicillin & $100(37 / 37)$ & $100(98 / 98)$ & - & - \\
\hline Fluoroquinolones & Enrofloxacin & $47(22 / 47)$ & $29(30 / 104)$ & 0.62 & 0.03 \\
\hline Lincosamides & Clindamycin & $87(41 / 47)$ & $91(95 / 104)$ & 1.05 & 0.43 \\
\hline Pleuromutilin & Tiamulin & $6(3 / 47)$ & $10(3 / 29)$ & 1.62 & 0.67 \\
\hline Sulfonamides & Sulfadimethoxine & $62(29 / 47)$ & $40(42 / 104)$ & 0.65 & 0.02 \\
\hline Potentiated sulfonamides & Trimethoprim-sulfamethoxazole & $32(15 / 47)$ & $19(20 / 104)$ & 0.60 & 0.09 \\
\hline \multirow{2}{*}{ Tetracyclines } & Chlortetracycline & $34(16 / 47)$ & $20(21 / 104)$ & 0.59 & 0.07 \\
\hline & Oxytetracycline & $64(30 / 47)$ & $85(88 / 104)$ & 1.33 & $<0.01$ \\
\hline
\end{tabular}

${ }^{1}$ Percentage represents resistance as a percentage of $B$. trehalosi isolates tested against that antibiotic during the 5-yr period.

${ }^{2} \mathrm{RR}=$ relative risk; if $\mathrm{RR}>1$, the likelihood of resistance in 2013-2017 is higher than the likelihood of resistance in 2008-2012. If $\mathrm{RR}<1$, the likelihood of resistance in 2013-2017 is lower than the likelihood of resistance in 2008-2012. No Clinical and Laboratory Standards Institute breakpoints for ceftiofur, danofloxacin, gamithromycin, tildipirosin, tulathromycin, or tylosin. 
use (Dowling, 2013). A common link among all 3 antibiotic classes is that they are commonly used as antimicrobial growth promotants in the swine and poultry industries (Marshall and Levy, 2011). When antibiotics are used for growth promotant purposes, they are usually added to feed or water at low, but continuous, dosing. This allows for the potential development of acquired resistance in the normal microflora (Marshall and Levy, 2011). These resistance genes not only spread through large numbers of animals receiving treatment, but may also cross into other species, such as cattle (Marshall and Levy, 2011).

For P. multocida, M. haemolytica, and H. somni isolates, resistance against the third-generation cephalosporin, ceftiofur, is rare. In response to increased resistance of gram-negative bacteria, such as Salmonella spp. and Escherichia coli, isolated from food animals in the United States, extralabel use of this drug with regard to dose, route, frequency of administration, or for disease prevention is strictly prohibited in food animals (Prescott, 2013a). In our study, the number of isolates resistant to ceftiofur remained unchanged following implementation of this regulation in 2012. In a study by Portis et al. (2012), ceftiofur remained very active against the same BRD pathogens from 2000 to 2009. Overall, resistance to the $\beta$-lactam class of antibiotics was minimal across all pathogens except $B$. trehalosi. Although B. trehalosi isolates only made up $4 \%$ of the total pathogens isolated during the study period, $100 \%$ of the isolates were resistant to penicillin.

The BRD isolates remained susceptible to florfenicol throughout the study period. Although a report has shown decreasing susceptibility to florfenicol across BRD pathogens (Portis et al., 2012), our study found that resistance has remained unchanged or decreased for all pathogens other than M. haemolytica.

A decrease $(P<0.05)$ in resistance to gentamicin was shown for $P$. multocida and B. trehalosi isolates. Most notably, a decrease $(P<0.05)$ in resistance occurred for all 3 antibiotics within the aminoglycoside class for B. trehalosi.

In our study, resistance to sulfonamides alone was higher than resistance to potentiated sulfonamides. Sulfadimethoxine is the only sulfonamide approved for use in lactating dairy cattle in the United States (Prescott, 2013b). Although resistance was high, our study revealed $(P<0.05)$ that for all pathogens, resistance to sulfadimethoxine was lower in the second 5-yr period.

Fluoroquinolones, like third-generation cephalosporins, are important antimicrobials in both human and veterinary medicine. For P. multocida, resistance against enrofloxacin is low $(<2 \%)$ and has not changed over the 10-yr study period. An increase has occurred in resistance against danofloxacin, which is only labeled for treatment of BRD associated with $M$. haemolytica and P. multocida in beef cattle. Histophilus somni and B. trehalosi have become less resistant $(P<0.05)$ to enrofloxacin. Of greater concern is the increased resistance $(P<0.05)$ of $M$. haemolytica to fluoroquinolones during the study period. A similar trend in resistance was seen in a study by Portis et al. (2012).

Increasing resistance of BRD pathogens to macrolides has been documented in numerous studies (Portis et al., 2012; Timsit et al., 2017). Over our 10-yr study period, resistance of $P$. multocida organisms against macrolides has remained unchanged, whereas resistance of $B$. trehalosi isolates to tilmicosin has decreased $(P<0.05)$. Our study showed an increase $(P<0.05)$ in resistance of $M$. haemolytica isolates to tilmicosin and tulathromycin. Resistance of $H$. somni isolates to macrolides remains static except for tylosin, to which $100 \%$ of isolates were resistant in the second 5 -yr period. Often, resistance genes against macrolides and tetracyclines are present in combination (Timsit et al., 2017). For the tetracyclines analyzed in our study, all pathogen groups had greater resistance against oxytetracycline than chlortetracycline. Resistance to oxytetracycline was moderate (27-36\% of isolates) for P. multocida, M. haemolytica, and H. somni. Our study found less resistance to oxytetracycline than what has been previously reported (Portis et al., 2012; Timsit et al., 2017). Although no appreciable changes occurred in the proportion of susceptible isolates for P. multocida, M. haemolytica, or H. somni, there was an increase $(P$ $<0.05)$ in resistance against oxytetracycline for $B$. trehalosi.

Our study has important limitations. Many samples sent to the WVDL are likely collected after antibiotic treatment has failed. A study by Magstadt et al. (2018) showed that prior antibiotic treatment was associated with an increased percentage of AMR in bacterial isolates from cases of BRD; therefore, our analysis may be biased toward a more resistant population than what would be found in the general population. Second, it is important to note that CLSI guidelines are the gold standard for determining a host-pathogen-drug combination breakpoint as those breakpoints are peer reviewed and updated periodically. Trek Diagnostic Systems (Cleveland, $\mathrm{OH}$ ) provides some breakpoints that are "supplied by veterinary customer," which are not peer reviewed. This study uses some breakpoints established by the veterinary customer to provide easier interpretation of the data and at times those breakpoints were then adopted by CLSI. Given the lack of CLSI interpretative criteria for certain host-pathogendrug combinations, namely for B. trehalosi, which had no CLSI established breakpoints, analysis was limited 
to applying breakpoints from other host-pathogen-drug combinations. Based on CLSI standards, these numbers are not interpretable, thus not allowing for precise conclusions to be made about the isolate's resistance.

The data in our analysis came from over 4,000 bovine isolates during a 10 -yr period. Although this is a substantial number, it only represents a small portion of the BRD pathogen population in the United States, which potentially decreases the external validity of our study. Although it is estimated that $>90 \%$ of isolates originated from cattle on dairy farms, exact breed information was not always available.

The strength of using interpretive criteria developed using CLSI breakpoints is that many of the factors related to the disease outcome, such as host response and bacterial resistance expression, are accounted for in the final interpretation (Lubbers and Turnidge, 2015). In our study, we analyzed interpretive criteria as a means of relating MIC data to an expected clinical outcome, but recognize that reporting the MIC frequency distributions would have provided more details of the dynamics of change in antibiotic susceptibility over time (Boerlin and White, 2013).

When we analyzed the proportion of resistant isolates for each pathogen-drug combination, we only included the resistant interpretation in our model (i.e., intermediate and susceptible isolates were not considered resistant). The CLSI definition of intermediate implies that a bacterial infection may be appropriately treated in body sites where the drugs are physiologically concentrated, or when a high dosage of drug can be used (CLSI, 2013). Our way of grouping the interpretative criteria may have led to an underestimation of percent resistance, especially for drugs that do not concentrate in the lung or when extralabel dosing is prohibited.

\section{CONCLUSIONS}

The current study provides a regional perspective on the proportion of common BRD pathogen isolates cultured from respiratory tract samples in cattle and describes changes in antibiotic resistance between two 5-yr time periods. Pasteurella multocida is commonly isolated from bovine respiratory samples and resistance is relatively low against the most commonly used antibiotics. In contrast, for M. haemolytica and $H$. somni, resistance against certain fluoroquinolone and macrolide class antibiotics has increased. Continued BRD bacterial pathogen surveillance and monitoring of antibiotic resistance in cattle on dairy farms are needed. However, these efforts will only help preserve the utility of antimicrobials if the information can be used to improve treatment efficacy. Future directions for this data could include evaluating whether there was an association between the type of respiratory sample evaluated and proportion of bacterial isolates cultured as well as the likelihood of AMR in pathogens isolated from antibiotic-treated versus untreated cattle.

\section{ACKNOWLEDGMENTS}

A special thanks to Erik Twaroski, from the bacteriology section at Wisconsin Veterinary Diagnostic Laboratory, for his assistance with data collection. The authors have not stated any conflicts of interest.

\section{REFERENCES}

Bach, A. 2011. Associations between several aspects of heifer development and dairy cow survivability to second lactation. J. Dairy Sci. 94:1052-1057. https://doi.org/10.3168/jds.2010-3633.

Boerlin, P., and D. G. White. 2013. Antimicrobial resistance and its epidemiology. Pages 21-36 in Antimicrobial Therapy in Veterinary Medicine: Fifth Edition. S. Giguére, J. F. Prescott, and P. M. Dowling, ed. Wiley-Blackwell, Danvers, MA.

CLSI. 2006. M100. Performance standards for antimicrobial susceptibility testing. Clinical and Laboratory Standards Institute, Wayne, PA.

CLSI. 2008. M31-A3. Performance standards for antimicrobial disk and dilution susceptibility tests for bacteria isolated from animals; Approved Standard. Clinical and Laboratory Standards Institute, Wayne, PA.

CLSI. 2013. M100-S23. Performance standards for antimicrobial disk and dilution susceptibility tests for bacteria isolated from animals; CLSI supplement. Clinical and Laboratory Standards Institute, Wayne, PA.

Confer, A. W. 2009. Update on bacterial pathogenesis in BRD. Anim. Health Res. Rev. 10:145-148. https://doi.org/10.1017/ S1466252309990193.

DeDonder, K. D., and M. D. Apley. 2015. A review of the expected effects of antimicrobials in bovine respiratory disease treatment and control using outcomes from published randomized clinical trials with negative controls. Vet. Clin. North Am. Food Anim. Pract. 31:97-111. https://doi.org/10.1016/j.cvfa.2014.11.003.

Dowling, P. M. 2013. Aminoglycosides and aminocyclitols. Page 243 in Antimicrobial Therapy in Veterinary Medicine: Fifth Edition. S. Giguére, J. F. Prescott, and P. M. Dowling, ed. Wiley-Blackwell, Danvers, MA.

Dunn, T. R., T. L. Ollivett, D. L. Renaud, K. E. Leslie, S. J. LeBlanc, T. F. Duffield, and D. F. Kelton. 2018. The effect of lung consolidation, as determined by ultrasonography, on first-lactation milk production in Holstein dairy calves. J. Dairy Sci. 101:5404-5410. https://doi.org/10.3168/jds.2017-13870.

Giguére, S. 2013a. Lincosamides, pleuromutilins, and streptogramins. Page 203 in Antimicrobial Therapy in Veterinary Medicine: Fifth Edition. S. Giguére, J. F. Prescott, and P. M. Dowling, ed. Blackwell, Danvers, MA.

Giguére, S. 2013b. Macrolides, azalides, and ketolides. Pages 211-218 in Antimicrobial Therapy in Veterinary Medicine: Fifth Edition. S. Giguére, J. F. Prescott, and P. M. Dowling, ed. Blackwell, Danvers, MA.

Lubbers, B. V., and J. Turnidge. 2015. Antimicrobial susceptibility testing for bovine respiratory disease: Getting more from diagnostic results. Vet. J. 203:149-154. https://doi.org/10.1016/j.tvjl .2014.12.009.

Magstadt, D. R., A. M. Schuler, J. F. Coetzee, A. C. Krull, A. M. O'Connor, V. L. Cooper, and T. J. Engelken. 2018. Treatment history and antimicrobial susceptibility results for Mannheimia haemolytica, Pasteurella multocida, and Histophilus somni isolates from bovine respiratory disease cases submitted to the Iowa State University Veterinary Diagnostic Laboratory from 2013 to 
2015. J. Vet. Diagn. Invest. 30:99-104. https://doi.org/10.1177/ 1040638717737589

Marshall, B. M., and S. B. Levy. 2011. Food animals and antimicrobials: Impacts on human health. Clin. Microbiol. Rev. 24:718-733. https://doi.org/10.1128/CMR.00002-11.

Panciera, R. J., and A. W. Confer. 2010. Pathogenesis and pathology of bovine pneumonia. Vet. Clin. North Am. Food Anim. Pract. 26:191-214. https://doi.org/10.1016/j.cvfa.2010.04.001.

Portis, E., C. Lindeman, L. Johansen, and G. Stoltman. 2012. A tenyear (2000-2009) study of antimicrobial susceptibility of bacteria that cause bovine respiratory disease complex-Mannheimia haemolytica, Pasteurella multocida, and Histophilus somni-in the United States and Canada. J. Vet. Diagn. Invest. 24:932-944. https://doi.org/10.1177/1040638712457559.

Prescott, J. F. 2013a. Beta-lactam antibiotics: Penam penicillins and cephalosporins. Pages 145 and 167 in Antimicrobial Therapy in Veterinary Medicine: Fifth Edition. S. Giguére, J. F. Prescott, and P. M. Dowling, ed. Wiley-Blackwell, Danvers, MA.

Prescott, J. F. 2013b. Sulfonamides, diaminopyrimidines, and their combinations. Page 285 in Antimicrobial Therapy in Veterinary Medicine: Fifth Edition. S. Giguére, J. F. Prescott, and P. M. Dowling, ed. Wiley-Blackwell, Danvers, MA.

Teixeira, A. G. V., J. A. McArt, and R. C. Bicalho. 2017. Thoracic ultrasound assessment of lung consolidation at weaning in Holstein dairy heifers: Reproductive performance and survival. J. Dairy Sci. 100:2985-2991. https://doi.org/10.3168/jds.2016-12016.

Timsit, E., J. Hallewell, C. Booker, N. Tison, S. Amat, and T. W. Alexander. 2017. Prevalence and antimicrobial susceptibility of Mannheimia haemolytica, Pasteurella multocida, and Histophilus somni isolated from the lower respiratory tract of healthy feedlot cattle and those diagnosed with bovine respiratory disease. Vet.
Microbiol. 208:118-125. https://doi.org/10.1016/j.vetmic.2017.07 .013 .

USDA. 2018. Dairy 2014, Health and management practices on U.S. dairy operations, 2014. USDA:APHIS:VS, CEAH Fort Collins, CO. \#696.0218

Valenzuela, J. R., A. K. Sethi, N. A. Aulik, and K. P. Poulsen. 2017. Antimicrobial resistance patterns of bovine Salmonella enterica isolates submitted to the Wisconsin Veterinary Diagnostic Laboratory: 2006-2015. J. Dairy Sci. 100:1319-1330. https://doi.org/10 $.3168 /$ jds.2016-11419.

Watts, J. L., and M. T. Sweeney. 2010. Antimicrobial resistance in bovine respiratory disease pathogens: Measures, trends, and impact on efficacy. Vet. Clin. North Am. Food Anim. Pract. 26:79-88. https://doi.org/10.1016/j.cvfa.2009.10.009.

Woolums, A. R. 2015. Ruminant respiratory system. Pages 583-603 in Large Animal Internal Medicine: Fifth Edition. B. P. Smith, ed. Elsevier, St. Louis, MO.

WHO (World Health Organization). 2019. Critically important antimicrobials for human medicine, 6th revision. Accessed Nov. 4 2019. https://apps.who.int/iris/bitstream/handle/10665/312266/ 9789241515528-eng.pdf?ua=1.

\section{ORCIDS}

C. L. Holschbach $\odot$ https://orcid.org/0000-0001-5173-5240

N. Aulik @ https://orcid.org/0000-0002-0388-5858

K. Poulsen (1) https://orcid.org/0000-0003-0302-254X

T. L. Ollivett $\odot$ https://orcid.org/0000-0003-2490-5835 